\title{
Efficient enumeration of rooted maps of a given orientable genus by number of faces and vertices
}

\author{
Timothy R. S. Walsh \\ Department of Computer Science, University of Quebec in Montreal (UQAM), \\ P.O. Box 8888, Station A, Montreal, Quebec, Canada, HC3-3P8 \\ Alain Giorgetti * \\ Department of Computer Science for Complex Systems, \\ FEMTO-ST Institute (UMR 6174), \\ University of Franche-Comté (UFC), 16 route de Gray, 25030 Besançon, France
}

Received 11 February 2011, accepted 1 March 2013, published online 7 May 2013

\begin{abstract}
We simplify the recurrence satisfied by the polynomial part of the generating function that counts rooted maps of positive orientable genus $g$ by number of vertices and faces. We have written an optimized program in $\mathrm{C}++$ for computing this generating function and constructing tables of numbers of rooted maps, and we describe some of these optimizations here. Using this program we extended the enumeration of rooted maps of orientable genus $g$ by number of vertices and faces to $g=4,5$ and 6 and by number of edges to $g=5$ and 6 and conjectured a further simplification of the generating function that counts rooted maps by number of edges. Our program is documented and available on request, allowing anyone with a sufficiently powerful computer to carry the calculations even further.
\end{abstract}

Keywords: Efficient enumeration, rooted maps, orientable genus, generating functions

Math. Subj. Class.: 05C30, 05A15

\section{Introduction: definitions and history}

A map is defined topologically as a 2-cell imbedding of a connected graph, loops and multiple edges allowed, in a 2-dimensional surface. The faces of a map are the connected components of the complement of the graph in the surface. In this article the surface is

\footnotetext{
* Corresponding author.

E-mail addresses: walsh.timothy@uqam.ca (Timothy R. S. Walsh), alain.giorgetti@femto-st.fr (Alain Giorgetti)
} 
assumed to be without boundary and orientable, with an orientation already attributed to it (clockwise, say), so that it is completely described by a non-negative integer $g$, its genus. For short, a map on a surface of genus $g$ will be called a genus-g map. A planar map is a genus-0 map (a map on a sphere) and a toroidal map is a genus-1 map (a map on a torus or donut). If a map on a surface of genus $g$ has $v$ vertices, $e$ edges and $f$ faces, then by the Euler-Poincaré formula [7, chap. 9]

$$
v-e+f=2(1-g) .
$$

Two maps are equivalent if there is an orientation-preserving homeomorphism between their imbedding surfaces that takes the vertices, edges and faces of one map into the vertices, edges and faces of the other. A dart of a map or graph is a semi-edge. A loop is assumed to be incident twice with the same vertex, so that every edge, whether or not it is a loop, is incident to two darts. The degree of a vertex is the number of darts incident to it. The face incident to a dart $d$ is the face incident to the edge containing $d$ and on the left of an observer on $d$ facing away from the vertex incident to $d$ and the degree of a face is the number of darts incident to it. A rooted map is a map with a distinguished dart, its root. Two rooted maps are equivalent if there is an orientation-preserving homeomorphism between their imbedding surfaces that takes the vertices, edges, faces and the root of one map into the vertices, edges, faces and the root of the other. A combinatorial map is a connected graph with a cyclic order imposed on the darts incident to each vertex, representing the order in which the darts of a (topological) map are encountered during a rotation around the vertex according to the orientation of the imbedding surface. The darts incident to a face are encountered by successive application of the following pair of actions: go from the current dart to the dart on the other end of the same edge and then to the next dart incident to the same vertex according to the cyclic order. In this way the faces of a combinatorial map can be counted, so that its genus can be calculated from (1.1). Two combinatorial maps are equivalent if they are related by a map isomorphism - a graph isomorphism that preserves this cyclic order - with an analogous definition for the equivalence of two rooted combinatorial maps.

It is worth noting that rooted combinatorial maps with $e$ edges are in one-to-one correspondence with torsion-free subgroups of index $2 e$ in the triangle group $\Delta(\infty, 2, \infty)=$ $\left\langle x, y, z \mid y^{2}=x y z=1\right\rangle \cong Z * Z_{2}$ [12]. Rooted combinatorial maps are indeed a permutation representation of these groups. Details about this correspondence may be found e.g. in [13].

By enumerating maps with a given set of properties, whether rooted or not, we mean counting the number of equivalence classes of maps with these properties. It was shown in [12] that each equivalence class of topological maps is uniquely defined by an equivalence class of combinatorial maps; so for the purposes of enumeration, the term "map" can be taken to mean "combinatorial map".

Let $m_{g}(v, f)$ be the number of rooted genus- $g$ maps with $v$ vertices and $f$ faces. By face-vertex duality, this number is equal to the number $m_{g}(f, v)$ of rooted genus- $g$ maps with $f$ vertices and $v$ faces. The generating function that counts rooted genus- $g$ maps is the following formal power series in two variables $u$ and $w$ :

$$
M_{g}(w, u)=\sum_{v, f \geq 1} m_{g}(v, f) w^{v} u^{f} .
$$

Rooted maps were introduced in [15] because they are easier to count than unrooted 
maps; this is because only the trivial map automorphism preserves the root [16], so that rooted maps can be counted without considering map automorphisms. In [15], W. T. Tutte found a closed-form formula for the number of rooted planar maps with $n$ edges. In [16], he found a parametric system of equations defining $M_{0}(w, u)$. In [1] D. Arquès obtained the simpler expression

$$
M_{0}(w, u)=p q(1-2 p-2 q)
$$

with the parameters $p$ and $q$ defined by

$$
w=p(1-p-2 q)
$$

and

$$
u=q(1-2 p-q),
$$

where $p=q=0$ when $w=u=0$. In [16], a recursive formula was found for the number of rooted planar maps given the number of vertices, the number of edges, and the degree of the face containing the root; these numbers of maps were then added over all possible degrees of this face and the result expressed in terms of generating functions. In [17], the first author generalized this method to obtain a recursive formula for the number of maps of genus $g$ with a distinguished dart in each vertex given the number of vertices and the degree of each one; these numbers were then multiplied by the appropriate factor and added over all possible non-increasing sequences of vertex-degrees summing to $2 e$ to obtain the number of rooted maps of genus $g$ with $e$ edges and $v$ vertices. A table of these numbers of maps with up to 14 edges appears in [17] (see [19] for a published account of this work and a table of maps with up to 11 edges) but no attempt was made there to express this result in terms of generating functions. We note here that a similar generalization in which the degrees of all the faces are known but only some of them have a distinguished edge on their boundary, and these faces must be of degree at least 3, appears in [8], where it is attributed to Tutte under the name of Tutte's recursion equations.

In [5] an improvement on the method of [17] was introduced: to count rooted genus$g$ maps it is sufficient to know the degree of the first $g+1$ vertices and to distinguish a dart of only the first vertex as the root, thus reducing the number of maps that have to be considered. Using doubly-rooted maps, D. Arquès [2] obtained the analogue of (1.3) for toroidal maps:

$$
M_{1}(w, u)=\frac{p q(1-p-q)}{\left[(1-2 p-2 q)^{2}-4 p q\right]^{2}} .
$$

From this result, he obtained a closed-form formula for the number of rooted toroidal maps with $e$ edges and another one for the number of rooted toroidal maps with $v$ vertices and $f$ faces. In [6] a generating function was obtained for the number of rooted maps of genus 2 and 3 with $e$ edges.

In [9] the second author generalized (1.6) and obtained a general form for the generating function $M_{g}(w, u)$ counting rooted maps of any genus $g>0$ :

$$
M_{g}(w, u)=\frac{p q(1-p-q) P_{g}(p, q)}{\left[(1-2 p-2 q)^{2}-4 p q\right]^{5 g-3}},
$$

where $P_{g}(p, q)$ is a symmetric polynomial in $p$ and $q$ of total degree bounded by $6 g-6$ with integral coefficients (in what follows, unless otherwise specified, all the polynomials defined here are polynomials in $p$ and $q$ ). 
Let us now briefly explain the interest of this result for rooted map counting. In [3, 9] the polynomials $P_{g}[p, q]$ were calculated for $g=2$ and 3 . In this article we extend this calculation to $g=4,5$ and 6 . To derive the generating function $M_{g}(w, u)$ from $P_{g}[p, q]$, we compute $p$ and $q$ as power series in $w$ and $u$ iteratively from (1.4) and (1.5) and then we replace $p$ and $q$ by their respective power series in (1.7) to obtain $M_{g}(w, u)$. By (1.2) the number of rooted maps of genus $g$ with $v$ vertices and $f$ faces is the coefficient of $w^{v} u^{f}$ in $M_{g}(w, u)$. In this way we enumerate rooted maps of genus 4, 5 and 6 with $v$ vertices and $f$ faces for any $v$ and $f$ greater than or equal to 1 .

The polynomial $P_{g}$ in (1.7) is defined in terms of another polynomial $T_{g}$ of degree bounded by $10 g-8$ by

$$
P_{g}=\frac{T_{g}}{(1-p)^{4 g-2}},
$$

and that polynomial, in turn, is defined in terms of a family of polynomials $R_{g}\left(n_{1}, \ldots, n_{r}\right)$ in $p$ and $q$ by

$$
T_{g}=R_{g-1}(0,0)+\sum_{j=1}^{g-1} q(1-p-q) R_{j}(0) R_{g-j}(0) .
$$

The degree of the polynomial $R_{g}\left(n_{1}, \ldots, n_{r}\right)$ is defined by the equation

$$
\operatorname{deg} R_{g}\left(n_{1}, \ldots, n_{r}\right)=2\left(n_{1}+\ldots+n_{r}\right)+7 r+10 g-12 .
$$

The polynomials $R_{g}\left(n_{1}, \ldots, n_{r}\right)$ are defined recursively in terms of several other families of polynomials and a recursively-defined family of rational functions of $p$ and $q$. We have two finite families of polynomials in $p$ alone defined by the following two sets of equations.

$$
\begin{gathered}
K_{0}(p)=-p ; \quad K_{1}(p)=-1-p ; \quad K_{2}(p)=-1 ; \\
K_{m}(p)=0 \text { for all } m \geq 3 . \\
L_{0}(p)=-p ; \quad L_{1}(p)=-1-2 p ; \\
L_{2}(p)=-2-p ; \quad L_{3}=-1 ; \\
L_{k}(p)=0 \text { for all } k \geq 4 .
\end{gathered}
$$

In what follows, the parameter $p$ will be omitted, so that these polynomials will be referred to as $K_{m}$ and $L_{k}$. We then have two polynomials $H$ and $J$ (in $p$ and $q$ ) defined by

$$
J=q(1-p-q)
$$

and

$$
H=(1-2 p-2 q)^{2}-4 p q .
$$

Finally we have an infinite family $\left(E_{k}\right)_{k \geq 1}$ of rational functions of $p$ and $q$, all but the first two of which are polynomials, defined recursively by

$$
\begin{gathered}
E_{1}=\frac{1}{2 J(1-p)^{2}} ; \\
E_{2}=\frac{-p-4 q+2 p^{2}+4 q^{2}+4 p q}{2 J(1-p)^{2}} ; \\
E_{k}=-J(1-p)^{2} \sum_{i=2}^{i=k-1} E_{i} E_{k+1-i} \text { for all } k \geq 4 .
\end{gathered}
$$


To make the recursive definition of the polynomials $R_{g}\left(n_{1}, \ldots, n_{r}\right)$ comprehensible, we first explain the abbreviations and conventions we use. For any positive integer $r,[r]$ denotes the sequence $(2, \ldots, r)$ if $r \geq 2$ and the empty sequence if $r=1$. For any subsequence $X$ of $[r],[r]-X$ denotes the subsequence of the elements of $[r]$ that are not in $X$. For any sequence $\left(n_{2}, \ldots, n_{r}\right)$ of integers, $N_{X}$ denotes the sequence of those $n_{i}$ such that $i$ is in $X$ and $N_{j}$ denotes the sequence $\left(n_{2}, \ldots, n_{j-1}, n_{j+1}, \ldots, n_{r}\right)$. By convention, a sum over an empty domain is equal to zero.

The polynomials $R_{0}\left(n_{1}\right)$ are not defined. The anchor of this recursive definition is

$$
R_{0}(0,0)=(1-p)^{2}
$$

If $g=0$ and $r=2$ but $\left(n_{1}, n_{2}\right) \neq(0,0)$, then we have

$$
\begin{aligned}
R_{0}\left(n_{1}, n_{2}\right)= & (1-p)^{2}\left(-n_{2} H E_{n_{1}+n_{2}+2}-\left(n_{2}+1\right) E_{n_{1}+n_{2}+3}\right) \\
& +2 J(1-p)^{2} \sum_{\substack{i+j+k=n_{1}+1 \\
i>0, k<n_{1}}}(-1)^{j+1} H^{j} E_{i} R_{0}\left(k, n_{2}\right) .
\end{aligned}
$$

We note that (1.16) is a special case of (1.17) where $n_{1}=n_{2}=0$.

If $(g, r) \neq(0,2)$, then

$$
R_{g}\left(n_{1}, \ldots, n_{r}\right)=\operatorname{term}_{1}+\operatorname{term}_{2}+\text { term }_{3}+\text { term }_{4},
$$

where

$$
\operatorname{term}_{1}=2 J(1-p)^{2} \sum_{\substack{i+j+k=n_{1}+1 \\ i>0, k<n_{1}}}(-1)^{j+1} H^{j} E_{i} R_{g}\left(k, n_{2}, \ldots, n_{r}\right)
$$

(we note that the second line of (1.17) is a special case of (1.19) where $g=0$ and $r=2$ ),

$$
\begin{gathered}
\operatorname{term}_{2}=J \sum_{\substack{k+l+m=n_{1}+1 \\
0 \leq j \leq g \\
X \subseteq[r] \\
(j, X) \neq(0, \emptyset) \\
(j, X) \neq(g,[r])}} K_{m} H^{m} R_{j}\left(k, N_{X}\right) R_{g-j}\left(l, N_{[r]-X}\right), \\
\operatorname{term}_{3}=\sum_{\substack{i+j+m=n_{1}+1 \\
i+j}} K_{m} H^{m} R_{g-1}\left(i, j, N_{[r]}\right)
\end{gathered}
$$

and

$$
\operatorname{term}_{4}=\sum_{j=2}^{r}\left(\begin{array}{l}
n_{j} \sum_{k+l=n_{1}+n_{j}+2} L_{k} H^{k+1} R_{g}\left(l, N_{j}\right) \\
+\left(n_{j}+1\right) \sum_{k+l=n_{1}+n_{j}+3} L_{k} H^{k} R_{g}\left(l, N_{j}\right)
\end{array}\right) .
$$

It was shown in [9] that each polynomial $R_{g}\left(n_{1}, \ldots, n_{r}\right)$ is symmetric in all its variables. This was made possible by distinguishing a dart incident to each of the vertices whose degree is considered, which increases the size of the coefficients but does not increase the number of polynomials that have to be calculated.

We note here that in the account of these results published in [3] formula (1.17) and the sum in (1.15) are missing; the formulas are presented correctly in [9]. At that time the second author, programming in Maple, calculated the polynomial $P_{g}$ and the generating 
function $M_{g}(w, u)$ for $g=2$ and $g=3$ (these results are published in [3]) and also computed the generating function that counts rooted maps of genus 4 by number of edges. This result was recently included in [13], where it was used to count both rooted and unrooted maps of genus 4 by number of edges.

Recently, the second author extended his enumeration results to genus 5. The first author, programming mainly in $\mathrm{C}$, optimized the calculation of the polynomials $R_{g}\left(n_{1}, \ldots\right.$, $n_{r}$ ) and thus extended the enumeration by number of vertices and faces, as well as by number of edges, to genus 6 . Although each author used a different algorithm and a different programming language, we both obtained the same answers, and the numbers of rooted maps we calculated agree with the tables in [17], providing evidence of the correctness of our results. An account of these extensions is given in Sections 2 and 3. Tables of rooted map numbers are given in Appendix A. A discussion of the enumeration of rooted genus$g$ maps by number of edges appears in Section 4 and the polynomial part of each of the corresponding generating functions appears in Appendix B. Finally, a discussion of some open problems appears in Section 5.

\section{Results from the Maple program}

A first version of the Maple code written in 1998 implemented recurrence relations between the rational functions introduced in [4] for the computation of the generating functions $M_{g}$. It was not designed for efficiency but for validating formulas from [4]. That code has also been used for validating the formulas from (1.2) through (1.22) for the first values of $g, r$ and $n_{1}, \ldots, n_{r}$ (these formulas were first obtained from a long computation that was done by hand and is thus error-prone). When executed in 1998 with Maple V for computing $M_{4}(w, u)$ that code ran into a fundamental limitation (wired into the Maple kernel) of a maximal number of 65,535 terms in any polynomial.

That old code has been recently replaced by a simpler code implementing directly the recursion between polynomials described by the formulas from (1.2) through (1.22). The code is short (less than 400 lines) and resembles the mathematical formulas as much as possible in order to detect errors. All the results obtained by this new code match known results in rooted map enumeration. For all these reasons, it can be considered as a reference for the debugging of optimized implementations.

With a personal computer running under Windows XP with an Intel Core 2 Duo CPU at $2.19 \mathrm{GHz}$ and $3.5 \mathrm{~Gb}$ of memory, and a Maple 14 release supporting larger objects, the next two generating functions $M_{4}(w, u)$ and $M_{5}(w, u)$ were successfully computed in 4 minutes and 5 hours, respectively. It was, however, not sensible to continue using this inefficient prototype for computing the next generating functions. A better idea was to write an independent implementation optimizing memory space and execution time.

\section{Optimizations and the $\mathrm{C}$ program}

Aside from the advantage in execution speed that $\mathrm{C}$ has over Maple, the first author optimized the calculation of the polynomials $R_{g}\left(n_{1}, \ldots, n_{r}\right)$. One of these optimizations was made possible by the following observation.

Proposition 3.1. For any $(g, r) \neq(0,1)$ and any sequence $n_{1}, \ldots, n_{r}$, the polynomial $R_{g}\left(n_{1}, \ldots, n_{r}\right)$ is divisible by $(1-p)^{2}$.

Proof. We use generalized induction on the degree of a polynomial of the form $R_{g}\left(n_{1}, \ldots\right.$, 
$\left.n_{r}\right)$, which we call an R-polynomial.

Basic step (degree 2). The only $R$-polynomial of degree 2 is $R_{0}(0,0)=(1-p)^{2}$ : see (1.16).

Induction step. Suppose that the degree $d$ of a given $R$-polynomial $R_{g}\left(n_{1}, \ldots, n_{r}\right)$, as defined by (1.10), is greater than 2 and that every $R$-polynomial of degree $<d$ is divisible by $(1-p)^{2}$. We show that $R_{g}\left(n_{1}, \ldots, n_{r}\right)$ is also divisible by $(1-p)^{2}$. Since every $R$-polynomial on the right side of equations (1.17), (1.19), (1.20), (1.21) and (1.22) is of degree $<d$, it follows from the induction hypothesis that each such polynomial is divisible by $(1-p)^{2}$. We examine each of these equations in turn.

Equation (1.17). The first line contains a factor $(1-p)^{2}$. The term $E_{n_{1}+n_{2}+3}$ is a polynomial for any non-negative $n_{1}$ and $n_{2}$. The term $E_{n_{1}+n_{2}+2}$ is a polynomial unless $n_{1}=n_{2}=0$, but in this case $E_{n_{1}+n_{2}+2}$ is multiplied by $n_{2}=0$; so the first line of (1.17) is divisible by $(1-p)^{2}$. In the second line, each term of the sum contains a polynomial $R_{0}\left(k, n_{2}\right)$, which, by the induction hypothesis, is divisible by $(1-p)^{2}$. This factor of $(1-p)^{2}$ could be cancelled by $E_{1}$ or $E_{2}$, but the sum is nevertheless a polynomial, and the factor $(1-p)^{2}$ by which the sum is multiplied ensures that the second line of (1.17) too is divisible by $(1-p)^{2}$; so the right side of $(1.17)$ is divisible by $(1-p)^{2}$.

Equation (1.19). By an argument similar to the one used for the second line of (1.17), the right side of $(1.19)$ is divisible by $(1-p)^{2}$.

Equations (1.20)-(1.22). Each term in the sum contains at least one $R$-polynomial that is divisible by $(1-p)^{2}$; so the right side of each of these equations is divisible by $(1-p)^{2}$. It follows from $(1.18)$ that $R_{g}\left(n_{1}, \ldots, n_{r}\right)$ is divisible by $(1-p)^{2}$, which completes the proof.

We now modify equations (1.8)-(1.10), and (1.16)-(1.22) in the light of Proposition 3.1. We introduce a new family of polynomials (which we call $S$-polynomials) defined by

$$
S_{g}\left(n_{1}, \ldots, n_{r}\right)=R_{g}\left(n_{1}, \ldots, n_{r}\right) /(1-p)^{2}
$$

and we also let

$$
U_{g}=T_{g} /(1-p)^{2} .
$$

Then $U_{g}$ is a polynomial of degree $10(g-1)$ and (1.8)-(1.10) become (3.3)-(3.5), respectively.

$$
\begin{gathered}
P_{g}=\frac{U_{g}}{(1-p)^{4 g-4}}, \\
U_{g}=S_{g-1}(0,0)+q(1-p-q)(1-p)^{2} \sum_{j=1}^{g-1} S_{j}(0) S_{g-j}(0) . \\
\operatorname{deg} S_{g}\left(n_{1}, \ldots, n_{r}\right)=2\left(n_{1}+\ldots+n_{r}\right)+7(r-2)+10 g .
\end{gathered}
$$

Also, (1.16)-(1.22) become (3.6)-(3.12), respectively.

$$
S_{0}(0,0)=1,
$$




$$
\begin{aligned}
S_{0}\left(n_{1}, n_{2}\right)= & \left(-n_{2} H E_{n_{1}+n_{2}+2}-\left(n_{2}+1\right) E_{n_{1}+n_{2}+3}\right) \\
& +2 J(1-p)^{2} \sum_{\substack{i+j+k=n_{1}+1 \\
i>0, k<n_{1}}}(-1)^{j+1} H^{j} E_{i} S_{0}\left(k, n_{2}\right) .
\end{aligned}
$$

If $(g, r) \neq(0,2)$, then

$$
S_{g}\left(n_{1}, \ldots, n_{r}\right)=\text { term }_{5}+\text { term }_{6}+\text { term }_{7}+\text { term }_{8},
$$

where

$$
\begin{aligned}
& \operatorname{term}_{5}=2 J(1-p)^{2} \sum_{\substack{i+j+k=n_{1}+1 \\
i>0, k<n_{1}}}(-1)^{j+1} H^{j} E_{i} S_{g}\left(k, n_{2}, \ldots, n_{r}\right), \\
& \operatorname{term}_{6}=J(1-p)^{2} \sum_{\substack{k+l+m=n_{1}+1 \\
0 \leq j \leq g \\
X \subseteq[r] \\
(j, X) \neq(0, \emptyset) \\
(j, X) \neq(g,[r])}} K_{m} H^{m} S_{j}\left(k, N_{X}\right) S_{g-j}\left(l, N_{[r]-X}\right), \\
& \operatorname{term}_{7}=\sum_{i+j+m=n_{1}+1} K_{m} H^{m} S_{g-1}\left(i, j, N_{[r]}\right)
\end{aligned}
$$

and

$$
\operatorname{term}_{8}=\sum_{j=2}^{r}\left(\begin{array}{l}
n_{j} \sum_{k+l=n_{1}+n_{j}+2} L_{k} H^{k+1} S_{g}\left(l, N_{j}\right) \\
+\left(n_{j}+1\right) \sum_{k+l=n_{1}+n_{j}+3} L_{k} H^{k} S_{g}\left(l, N_{j}\right)
\end{array}\right) .
$$

Since $R_{g}\left(n_{1}, \ldots, n_{r}\right)$ is symmetric in all its variables, so is $S_{g}\left(n_{1}, \ldots, n_{r}\right)$; so only those polynomials $S_{g}\left(n_{1}, \ldots, n_{r}\right)$ with $n_{1} \leq \ldots \leq n_{r}$ are treated. In all the $S$-polynomials on the right side of each of the equations (3.9)-(3.12), only the first two variables can violate these inequalities; so they are inserted into their proper slots among the remaining variables to preserve the inequalities. Also, equation (3.4) is symmetric in $j$ and $g-j$, equation (3.10) is symmetric in $k$ and $l$ and equation (3.11) is symmetric in $i$ and $j$; so the calculations there can be cut almost in half. In equation (3.12), each polynomial $S_{g}\left(l, N_{j}\right)$ is calculated only once and then used twice. The following easily proved observations can be used to avoid calculating a polynomial that is identically 0 : term ${ }_{5}=0$ if $n_{1}=0$, term $_{6}=0$ if $g+r \leq 2$, term $7=0$ if $g=0$, term $8=0$ if $r=1$ or $(g, r)=(0,2)$. From these observations, it follows that the only term that could possibly contribute to $S_{0}\left(n_{1}\right)$ is term ${ }_{5}$. From (3.9) it follows by generalized induction on $n_{1}$ that $S_{0}\left(n_{1}\right)=0$ for all $n_{1} \geq 0$; so these polynomials do not have to be defined.

All the $S$-polynomials are stored in a single one-dimensional array $s$. A preliminary recursion does not calculate any of these polynomials. Instead, it calculates all the quadruples $(d, g, r, c)$ of parameters of the $S$-polynomials that will later be calculated, where $d=\operatorname{deg} S_{g}\left(n_{1}, \ldots, n_{r}\right)$ and $c$ is an integer coding the sequence $\left(n_{1}, \ldots, n_{r}\right)$, and stores the list of quadruples in four parallel arrays, one array for each of the four parameters $d$, $g, r, c$ and one element of all four arrays for each quadruple $(d, g, r, c)$. The program then sorts the four parallel arrays by degree $d$ using bucket sort, computes the number of $S$ polynomials that have to be calculated and the total number of terms in these polynomials and stores in two arrays the index in $s$ and the one in the four parallel arrays of the first term for each degree $d$. Then the $S$-polynomials are calculated in increasing order of their 
degree and stored in $s$. This can be done non-recursively because all the $S$-polynomials that need to be used will have already been stored and need only be found by searching the four parallel arrays, starting with the first index for the appropriate degree $d$, for the appropriate parameters, and adding $(d+1)(d+2) / 2$ to the index in $s$ each time the index in the four parallel arrays is increased by 1 . Once the last polynomial $S_{g-1}(0,0)$ has been calculated, first (3.4) is used to calculate $U_{g}$ and then (3.3) is used to calculate $P_{g}$ and its coefficients are stored in a text file, which is available from the first author on request.

The number of $S$-polynomials that have to be calculated is roughly the total number of partitions of all the positive integers up to $10(g-1)$. For each of these polynomials, the most expensive calculation is term 6 , because the sum there runs over all the partitions of the sequence $[r]=(2, \ldots, r)$, where $r$ can be as great as $g+1$, and involves multiplying two $S$-polynomials. The time-complexity of calculating $P_{g}$ is therefore exponential in $g$, but the optimizations made here nevertheless made it possible to calculate $P_{g}$ for a greater value of $g$ than was possible previously. Another program computes a table of numbers of rooted genus- $g$ maps counted by number of vertices and faces by reading this file and using (1.2) if $g \geq 1$ or (1.3) if $g=0$. Tables of numbers of rooted genus- $g$ maps for any $g \leq 6$ and with up to any reasonable number of edges are available from the first author on request.

The programs were written mainly in $\mathrm{C}$. The one that computes the polynomials is about 2000 lines long and the one that computes the tables is about 300 lines long. They both use the $\mathrm{C}++$ library CLN to do arithmetic on big integers because CLN reads arithmetic expressions in $\mathrm{C}$ that use only addition, multiplication and subtraction; only statements involving quotients, remainders, input/output of big integers and file management had to be modified. Since CLN requires a GNU compiler, XCODE was downloaded and installed by Jerome Tremblay, a computer technician at UQAM, who also downloaded and installed CLN and wrote sample $\mathrm{C}++$ statements for input/output of big numbers and file management.

The programs were executed on a 2004 Macintosh GR4 computer. The time taken to compute the polynomial $P_{g}$ varied from run to run. In Table 1 we show, for each $g$ from 1 to 6 , the number of $S$-polynomials that were calculated, the total number of terms in all these $S$-polynomials, and a typical execution time. Once the $S$-polynomials had been computed and stored, it took the computer only 48 seconds to make a table of numbers of genus- 6 maps with up to 42 edges counted by number of vertices and faces.

Source codes for both programs are included in release 0.3.2 and higher of the MAP project [11]. The polynomials $P_{2}$ and $P_{3}$ appear in [3]. The polynomials $P_{4}, P_{5}$ and $P_{6}$ are too large to be reproduced here. The coefficients of the polynomials $P_{g}[p, q]$ for $1 \leq g \leq 6$ are included in the MAP project and available from the first author on request. For $1 \leq g \leq 6$ tables of numbers of rooted genus- $g$ maps up to 20 edges are given in Appendix A. More numbers of rooted genus- $g$ maps for any $g \leq 6$ and with up to any reasonable number of edges can be obtained from code included in the MAP project and are available from the first author on request.

\section{Counting by number of edges}

To compute the generating function $M_{g}(z)=z^{2 g-2} M_{g}(z, z)$ that counts rooted genus- $g$ maps by number of edges alone, we use the substitution obtained in [13], which is a more compact form of the one obtained in [9] and published in [3]. Let

$$
p=q=m,
$$




\begin{tabular}{r|r|r|r}
$g$ & number of $S$-polynomials & total number of terms & execution time \\
\hline 1 & 1 & 1 & instantaneous \\
2 & 16 & 507 & 1 second \\
3 & 67 & 7407 & 10 seconds \\
4 & 205 & 49796 & 2 minutes \\
5 & 543 & 235410 & 20 minutes \\
6 & 1314 & 900114 & 3.5 hours
\end{tabular}

Table 1: Evaluation of the computation cost

where

$$
z=m(1-3 m) \text { and } m=0 \text { when } z=0 .
$$

By substituting from (4.1) into (1.4) and (1.5) to express $w$ and $u$ in terms of $m$ and then substituting into (1.7), we obtain the following equation for $g \geq 1$ :

$$
M_{g}(z)=\frac{m^{2 g}(1-3 m)^{2 g-2} P_{g}(m, m)}{(1-6 m)^{5 g-3}(1-2 m)^{5 g-4}} .
$$

For $g=0$, we substitute into (1.3) instead of (1.7) and obtain

$$
M_{0}(z)=(1-3 m)^{-2}(1-4 m) .
$$

The first author computed $M_{g}(z)$ from the computed values of $M_{g}(w, u)$ for $g \leq 6$. The program divides the polynomial $P_{g}(m, m)$ by $1-2 m$ as often as possible. The program then divides the resulting polynomial by 2 and by 3 as often as possible, extracts the appropriate constant factor and then stores the resulting generating function in another text file, also available from the first author. The second author computed $M_{g}(z)$ directly for $g \leq 6$. We then compared our formulas and verified that they agree. The formulas for $P_{g}(m, m)$ for $1 \leq g \leq 6$ appear in Appendix B. Now $P_{g}(m, m)$ is of degree $6 g-6$, but we found experimentally in 2009 that for $1 \leq g \leq 6, P_{g}(m, m)$ is divisible by $(1-2 m)^{2 g-2}$, so that the quotient is only of degree $4 g-4$, and we conjectured that this is the case for any positive integer $g$. In 2010, the second author proved that conjecture [10].

\section{Some interesting open problems}

The recurrences satisfied by the $R$ - and $S$-polynomials both result from proofs by induction. After the right conjecture has been guessed by observing the first computed terms, these proofs are not difficult to find, but they are tedious and error-prone due to the length of the expressions involved. Thus they are good candidates for automation. We plan to develop a suitable formal framework for assisting this kind of proofs with a computer algebra system. The challenge is to shorten the chain of conjectures and proofs about the general pattern of generating functions for counting rooted maps.

Once the numbers of rooted maps of genus up to $g$ are known, the number of unrooted maps up to genus $g$ can be calculated using the methods presented in [14]. As was mentioned above, the second author collaborated with A. Mednykh to count rooted and unrooted maps of genus 4 by number of edges [13]. It would be interesting to count unrooted genus- $g$ maps by number of vertices and faces for as many values of $g$ as possible (see [18] for an account of the progress made on this problem). 


\section{Acknowledgments}

The authors are grateful to Roman Nedela, Alexander Mednykh and the anonymous referees for helpful comments and suggestions.

\section{References}

[1] D. Arquès, Une relation fonctionnelle nouvelle sur les cartes planaires pointées, J. Comb. Theory B 39 (1985), 27-42.

[2] D. Arquès, Relations fonctionnelles et dénombrement des cartes pointées sur le tore, J. Comb. Theory B 43 (1987), 253-274.

[3] D. Arquès and A. Giorgetti, Énumération des cartes pointées de genre quelconque en fonction des nombres de sommets et de faces, J. Comb. Theory B 77 (1999), 1-24.

[4] D. Arquès and A. Giorgetti, Counting rooted maps on a surface, Theor. Comput. Sci. 234 (2000), 255-272.

[5] E. A. Bender and E. R. Canfield, The asymptotic number of rooted maps on a surface, J. Comb. Theory A 43 (1986), 244-257.

[6] E. A. Bender and E. R. Canfield, The number of rooted maps on an orientable surface, J. Comb. Theory B 53 (1991), 293-299.

[7] H. S. M. Coxeter, Regular Polytopes, 3rd ed., Dover, New York, 1973.

[8] B. Eynard and N. S. Orantin, Topological recursion in enumerative geometry and random matrices, J. Phys. A-Math. Theor. 42 (2009), 293001.

[9] A. Giorgetti, Combinatoire bijective et énumérative des cartes pointées sur une surface, $\mathrm{PhD}$ thesis, Université de Marne-la-Vallée, Institut Gaspard Monge, 1998, http://tel. archives-ouvertes.fr/tel-00724977.

[10] A. Giorgetti, Guessing a Conjecture in Enumerative Combinatorics and Proving It with a Computer Algebra System, in: T. Jebelean, M. Mosbah and N. Popov (eds.), SCSS'10, July 2010, pp. 5-18.

[11] A. Giorgetti, MAP project release 0.3.2, https://sourceforge.net/projects/ combi/files/map/, January 2013.

[12] G. A. Jones and D. Singerman, Theory of maps on orientable surfaces, Proc. London Math. Soc. 37 (1978), 273-307.

[13] A. Mednykh and A. Giorgetti, Enumeration of genus four maps by number of edges, Ars Math. Contemp. 4 (2011), 351-361.

[14] A. Mednykh and R. Nedela, Enumeration of unrooted maps of a given genus, J. Comb. Theory $B 96$ (2006), 706-729.

[15] W. T. Tutte, A census of planar maps, Canad. J. Math. 15 (1963), 249-271.

[16] W. T. Tutte. On the enumeration of planar maps. Bull. Amer. Math. Soc. 74 (1968), 64-74.

[17] T. R. S. Walsh, Combinatorial enumeration of non-planar maps, $\mathrm{PhD}$ thesis, University of Toronto, 1971.

[18] T. R. S. Walsh, A. Giorgetti and A. Mednykh, Enumeration of unrooted orientable maps of arbitrary genus by number of edges and vertices, Discrete Math. 312 (2012), 2660-2671.

[19] T. R. S. Walsh and A. B. Lehman, Counting rooted maps by genus I, J. Comb. Theory B 13 (1972), 192-218. 


\section{Appendix A}

Number $m_{g}(v, f)$ of rooted maps of genus $g$ with $e$ edges and $v$ vertices, for $1 \leq g \leq 6$ and $2 g \leq e \leq 20$. Lines whose column of $v$ is empty give the total number $m_{g}(e)$ of rooted maps of genus $g$ with $e$ edges.

\begin{tabular}{|c|c|c|c|c|}
\hline$e$ & $v$ & $g=1$ & $g=2$ & $g=3$ \\
\hline 2 & 1 & 1 & & \\
\hline 2 & & 1 & & \\
\hline 3 & 1 & 10 & & \\
\hline 3 & 2 & 10 & & \\
\hline 3 & & 20 & & \\
\hline 4 & 1 & 70 & 21 & \\
\hline 4 & 2 & 167 & & \\
\hline 4 & 3 & 70 & & \\
\hline 4 & & 307 & 21 & \\
\hline 5 & 1 & 420 & 483 & \\
\hline 5 & 2 & 1720 & 483 & \\
\hline 5 & 3 & 1720 & & \\
\hline 5 & 4 & 420 & & \\
\hline 5 & & 4280 & 966 & \\
\hline 6 & 1 & 2310 & 6468 & 1485 \\
\hline 6 & 2 & 14065 & 15018 & \\
\hline 6 & 3 & 24164 & 6468 & \\
\hline 6 & 4 & 14065 & & \\
\hline 6 & 5 & 2310 & & \\
\hline 6 & & 56914 & 27954 & 1485 \\
\hline 7 & 1 & 12012 & 66066 & 56628 \\
\hline 7 & 2 & 100156 & 258972 & 56628 \\
\hline 7 & 3 & 256116 & 258972 & \\
\hline 7 & 4 & 256116 & 66066 & \\
\hline 7 & 5 & 100156 & & \\
\hline 7 & 6 & 12012 & & \\
\hline 7 & & 736568 & 650076 & 113256 \\
\hline 8 & 1 & 60060 & 570570 & 1169740 \\
\hline 8 & 2 & 649950 & 3288327 & 2668750 \\
\hline 8 & 3 & 2278660 & 5554188 & 1169740 \\
\hline 8 & 4 & 3392843 & 3288327 & \\
\hline 8 & 5 & 2278660 & 570570 & \\
\hline 8 & 6 & 649950 & & \\
\hline 8 & 7 & 60060 & & \\
\hline 8 & & 9370183 & 13271982 & 5008230 \\
\hline 9 & 1 & 291720 & 4390386 & 17454580 \\
\hline 9 & 2 & 3944928 & 34374186 & 66449432 \\
\hline 9 & 3 & 17970784 & 85421118 & 66449432 \\
\hline 9 & 4 & 36703824 & 85421118 & 17454580 \\
\hline 9 & 5 & 36703824 & 34374186 & \\
\hline 9 & 6 & 17970784 & 4390386 & \\
\hline 9 & 7 & 3944928 & & \\
\hline 9 & 8 & 291720 & & \\
\hline 9 & & 117822512 & 248371380 & 167808024 \\
\hline 10 & 1 & 1385670 & 31039008 & 211083730 \\
\hline
\end{tabular}




\begin{tabular}{|c|c|c|c|c|}
\hline$e$ & $v$ & $g=1$ & $g=2$ & $g=3$ \\
\hline 10 & 2 & 22764165 & 313530000 & 1171704435 \\
\hline 10 & 3 & 129726760 & 1059255456 & 1955808460 \\
\hline 10 & 4 & 344468530 & 1558792200 & 1171704435 \\
\hline 10 & 5 & 472592916 & 1059255456 & 211083730 \\
\hline 10 & 6 & 344468530 & 313530000 & \\
\hline 10 & 7 & 129726760 & 31039008 & \\
\hline 10 & 8 & 22764165 & & \\
\hline 10 & 9 & 1385670 & & \\
\hline 10 & & 1469283166 & 4366441128 & 4721384790 \\
\hline 11 & 1 & 6466460 & 205633428 & 2198596400 \\
\hline 11 & 2 & 126264820 & 2583699888 & 16476937840 \\
\hline 11 & 3 & 875029804 & 11270290416 & 40121261136 \\
\hline 11 & 4 & 2908358552 & 22555934280 & 40121261136 \\
\hline 11 & 5 & 5188948072 & 22555934280 & 16476937840 \\
\hline 11 & 6 & 5188948072 & 11270290416 & 2198596400 \\
\hline 11 & 7 & 2908358552 & 2583699888 & \\
\hline 11 & 8 & 875029804 & 205633428 & \\
\hline 11 & 9 & 126264820 & & \\
\hline 11 & 10 & 6466460 & & \\
\hline 11 & & 18210135416 & 73231116024 & 117593590752 \\
\hline 12 & 1 & 29745716 & 1293938646 & 20465052608 \\
\hline 12 & 2 & 678405090 & 19678611645 & 196924458720 \\
\hline 12 & 3 & 5593305476 & 106853266632 & 647739636160 \\
\hline 12 & 4 & 22620890127 & 276221817810 & 945068384880 \\
\hline 12 & 5 & 50534154408 & 375708427812 & 647739636160 \\
\hline 12 & 6 & 65723863196 & 276221817810 & 196924458720 \\
\hline 12 & 7 & 50534154408 & 106853266632 & 20465052608 \\
\hline 12 & 8 & 22620890127 & 19678611645 & \\
\hline 12 & 9 & 5593305476 & 1293938646 & \\
\hline 12 & 10 & 678405090 & & \\
\hline 12 & 11 & 29745716 & & \\
\hline 12 & & 224636864830 & 1183803697278 & 2675326679856 \\
\hline 13 & 1 & 135207800 & 7808250450 & 174437377400 \\
\hline 13 & 2 & 3550829360 & 140725699686 & 2079913241120 \\
\hline 13 & 3 & 34225196720 & 925572602058 & 8789123742880 \\
\hline 13 & 4 & 164767964504 & 2979641557620 & 17326957790896 \\
\hline 13 & 5 & 448035881592 & 5235847653036 & 17326957790896 \\
\hline 13 & 6 & 729734918432 & 5235847653036 & 8789123742880 \\
\hline 13 & 7 & 729734918432 & 2979641557620 & 2079913241120 \\
\hline 13 & 8 & 448035881592 & 925572602058 & 174437377400 \\
\hline 13 & 9 & 164767964504 & 140725699686 & \\
\hline 13 & 10 & 34225196720 & 7808250450 & \\
\hline 13 & 11 & 3550829360 & & \\
\hline 13 & 12 & 135207800 & & \\
\hline 13 & & 2760899996816 & 18579191525700 & 56740864304592 \\
\hline 14 & 1 & 608435100 & 45510945480 & 1384928666550 \\
\hline 14 & 2 & 18182708362 & 955708437684 & 19925913354061 \\
\hline 14 & 3 & 201976335288 & 7454157823560 & 104395235785256 \\
\hline 14 & 4 & 1137369687454 & 29079129795702 & 264477214235234 \\
\hline 14 & 5 & 3682811916980 & 63648856688592 & 357391270819604 \\
\hline 14 & 6 & 7302676928666 & 82234427131416 & 264477214235234 \\
\hline 14 & 7 & 9145847808784 & 63648856688592 & 104395235785256 \\
\hline 14 & 8 & 7302676928666 & 29079129795702 & 19925913354061 \\
\hline 14 & 9 & 3682811916980 & 7454157823560 & 1384928666550 \\
\hline 14 & 10 & 1137369687454 & 955708437684 & \\
\hline 14 & 11 & 201976335288 & 45510945480 & \\
\hline 14 & 12 & 18182708362 & & \\
\hline 14 & 13 & 608435100 & & \\
\hline 14 & & 33833099832484 & 284601154513452 & 1137757854901806 \\
\hline 15 & 1 & 2714556600 & 257611421340 & 10369994005800 \\
\hline
\end{tabular}




\begin{tabular}{|c|c|c|c|c|}
\hline$e$ & $v$ & $g=1$ & $g=2$ & $g=3$ \\
\hline 15 & 2 & 91392185080 & 6216591472728 & 176357530955320 \\
\hline 15 & 3 & 1156128848680 & 56532447160536 & 1115525500250760 \\
\hline 15 & 4 & 7506901051000 & 261637840342860 & 3505018618003600 \\
\hline 15 & 5 & 28442316247080 & 694146691745820 & 6087558311398000 \\
\hline 15 & 6 & 67173739068760 & 1117259292848016 & 6087558311398000 \\
\hline 15 & 7 & 102432266545800 & 1117259292848016 & 3505018618003600 \\
\hline 15 & 8 & 102432266545800 & 694146691745820 & 1115525500250760 \\
\hline 15 & 9 & 67173739068760 & 261637840342860 & 176357530955320 \\
\hline 15 & 10 & 28442316247080 & 56532447160536 & 10369994005800 \\
\hline 15 & 11 & 7506901051000 & 6216591472728 & \\
\hline 15 & 12 & 1156128848680 & 257611421340 & \\
\hline 15 & 13 & 91392185080 & & \\
\hline 15 & 14 & 2714556600 & & \\
\hline 15 & & 413610917006000 & 4272100949982600 & 21789659909226960 \\
\hline 16 & 1 & 12021607800 & 1422156202740 & 73920866362200 \\
\hline 16 & 2 & 452077562620 & 38985279745230 & 1461629029629340 \\
\hline 16 & 3 & 6447533938280 & 407653880116680 & 10933959720960760 \\
\hline 16 & 4 & 47700234551918 & 2200626948631386 & 41491242915292306 \\
\hline 16 & 5 & 208462422428152 & 6928413234959820 & 89390908732820144 \\
\hline 16 & 6 & 576218752277476 & 13518984452463630 & 114899070275212424 \\
\hline 16 & 7 & 1046677747672360 & 16842445235560944 & 89390908732820144 \\
\hline 16 & 8 & 1274461449989715 & 13518984452463630 & 41491242915292306 \\
\hline 16 & 9 & 1046677747672360 & 6928413234959820 & 10933959720960760 \\
\hline 16 & 10 & 576218752277476 & 2200626948631386 & 1461629029629340 \\
\hline 16 & 11 & 208462422428152 & 407653880116680 & 73920866362200 \\
\hline 16 & 12 & 47700234551918 & 38985279745230 & \\
\hline 16 & 13 & 6447533938280 & 1422156202740 & \\
\hline 16 & 14 & 452077562620 & & \\
\hline 16 & 15 & 12021607800 & & \\
\hline 16 & & 5046403030066927 & 63034617139799916 & 401602392805341924 \\
\hline 17 & 1 & 52895074320 & 7683009544980 & 505297829133240 \\
\hline 17 & 2 & 2205359390592 & 236923660397172 & 11460411934448048 \\
\hline 17 & 3 & 35155923872640 & 2815913391715452 & 99727841192820016 \\
\hline 17 & 4 & 293370096957504 & 17486142956133684 & 447708887118504600 \\
\hline 17 & 5 & 1461307573813824 & 64232028100704156 & 1165172136542282424 \\
\hline 17 & 6 & 4660202610532480 & 148755268498286436 & 1857975645023518752 \\
\hline 17 & 7 & 9908748651241088 & 224686278407291148 & 1857975645023518752 \\
\hline 17 & 8 & 14373136466094880 & 224686278407291148 & 1165172136542282424 \\
\hline 17 & 9 & 14373136466094880 & 148755268498286436 & 447708887118504600 \\
\hline 17 & 10 & 9908748651241088 & 64232028100704156 & 99727841192820016 \\
\hline 17 & 11 & 4660202610532480 & 17486142956133684 & 11460411934448048 \\
\hline 17 & 12 & 1461307573813824 & 2815913391715452 & 505297829133240 \\
\hline 17 & 13 & 293370096957504 & 236923660397172 & \\
\hline 17 & 14 & 35155923872640 & 7683009544980 & \\
\hline 17 & 15 & 2205359390592 & & \\
\hline 17 & 16 & 52895074320 & & \\
\hline 17 & & 61468359153954656 & 916440476048146056 & 7165100439281414160 \\
\hline 18 & 1 & 231415950150 & 40729207226400 & 3331309741059300 \\
\hline 18 & 2 & 10627956019245 & 1401097546161936 & 85694099173907510 \\
\hline 18 & 3 & 187959014565840 & 18743188498056288 & 855779329367736840 \\
\hline 18 & 4 & 1753945289216484 & 132344695964811720 & 4470547991985864322 \\
\hline 18 & 5 & 9857665477085832 & 559373367462490656 & 13767319160210071404 \\
\hline 18 & 6 & 35839052357422132 & 1511718920778951024 & 26522236056202555206 \\
\hline 18 & 7 & 87930943305742512 & 2710382626755160416 & 32904419378927915376 \\
\hline 18 & 8 & 149314477245194262 & 3286157560248860532 & 26522236056202555206 \\
\hline 18 & 9 & 177882700353757460 & 2710382626755160416 & 13767319160210071404 \\
\hline 18 & 10 & 149314477245194262 & 1511718920778951024 & 4470547991985864322 \\
\hline 18 & 11 & 87930943305742512 & 559373367462490656 & 855779329367736840 \\
\hline 18 & 12 & 35839052357422132 & 132344695964811720 & 85694099173907510 \\
\hline 18 & 13 & 9857665477085832 & 18743188498056288 & 3331309741059300 \\
\hline 18 & 14 & 1753945289216484 & 1401097546161936 & \\
\hline 18 & 15 & 187959014565840 & 40729207226400 & \\
\hline 18 & 16 & 10627956019245 & & \\
\hline 18 & 17 & 231415950150 & & \\
\hline
\end{tabular}




\begin{tabular}{|c|c|c|c|c|}
\hline$e$ & $v$ & $g=1$ & $g=2$ & $g=3$ \\
\hline 18 & & 747672504476150374 & 13154166812674577412 & 124314235272290304540 \\
\hline 19 & 1 & 1007340018300 & 212347275857640 & 21280393666593600 \\
\hline 19 & 2 & 50668344988068 & 8089830217844928 & 614960028331370816 \\
\hline 19 & 3 & 987658610225052 & 120789163612555200 & 6968569097113244096 \\
\hline 19 & 4 & 10229201477344752 & 960323177351524512 & 41790549086980226368 \\
\hline 19 & 5 & 64309102366765200 & 4616545437250956192 & 149789855223187292608 \\
\hline 19 & 6 & 263868150558327376 & 14358354462488121408 & 341505418008822731328 \\
\hline 19 & 7 & 738178726378902064 & 30044423965980553536 & 511895831411154922176 \\
\hline 19 & 8 & 1446563778096423816 & 43241609165618454096 & 511895831411154922176 \\
\hline 19 & 9 & 2017523504473479992 & 43241609165618454096 & 341505418008822731328 \\
\hline 19 & 10 & 2017523504473479992 & 30044423965980553536 & 149789855223187292608 \\
\hline 19 & 11 & 1446563778096423816 & 14358354462488121408 & 41790549086980226368 \\
\hline 19 & 12 & 738178726378902064 & 4616545437250956192 & 6968569097113244096 \\
\hline 19 & 13 & 263868150558327376 & 960323177351524512 & 614960028331370816 \\
\hline 19 & 14 & 64309102366765200 & 120789163612555200 & 21280393666593600 \\
\hline 19 & 15 & 10229201477344752 & 8089830217844928 & \\
\hline 19 & 16 & 987658610225052 & 212347275857640 & \\
\hline 19 & 17 & 50668344988068 & & \\
\hline 19 & 18 & 1007340018300 & & \\
\hline 19 & & 9083423595292949240 & 186700695099591735024 & 2105172926498512761984 \\
\hline 20 & 1 & 4365140079300 & 1090848505817070 & 132216351453357600 \\
\hline 20 & 2 & 239250231713210 & 45732525474843801 & 4257157940494918160 \\
\hline 20 & 3 & 5110652802256260 & 756589971284883792 & 54217755730994858080 \\
\hline 20 & 4 & 58364244137596695 & 6716133365837116980 & 369061676845849000520 \\
\hline 20 & 5 & 407372683115470800 & 36362952155187558600 & 1518921342035154605600 \\
\hline 20 & 6 & 1870153808268516280 & 128656798319026864068 & 4031165546220945277040 \\
\hline 20 & 7 & 5905479331377981200 & 309859885439753598768 & 7151648337964982801760 \\
\hline 20 & 8 & 13196809961724011350 & 520516978029736518606 & 8640883781524178188980 \\
\hline 20 & 9 & 21241931655650633720 & 617910462111714896820 & 7151648337964982801760 \\
\hline 20 & 10 & 24868664942648145372 & 520516978029736518606 & 4031165546220945277040 \\
\hline 20 & 11 & 21241931655650633720 & 309859885439753598768 & 1518921342035154605600 \\
\hline 20 & 12 & 13196809961724011350 & 128656798319026864068 & 369061676845849000520 \\
\hline 20 & 13 & 5905479331377981200 & 36362952155187558600 & 54217755730994858080 \\
\hline 20 & 14 & 1870153808268516280 & 6716133365837116980 & 4257157940494918160 \\
\hline 20 & 15 & 407372683115470800 & 756589971284883792 & 132216351453357600 \\
\hline 20 & 16 & 58364244137596695 & 45732525474843801 & \\
\hline 20 & 17 & 5110652802256260 & 1090848505817070 & \\
\hline 20 & 18 & 239250231713210 & & \\
\hline 20 & 19 & 4365140079300 & & \\
\hline 20 & & 110239596847544663002 & 2623742783421329300190 & 34899691847703927826500 \\
\hline
\end{tabular}




\begin{tabular}{|c|c|c|c|c|}
\hline$e$ & $v$ & $g=4$ & $g=5$ & $g=6$ \\
\hline 8 & 1 & 225225 & & \\
\hline 8 & & 225225 & & \\
\hline 9 & 1 & 12317877 & & \\
\hline 9 & 2 & 12317877 & & \\
\hline 9 & & 24635754 & & \\
\hline 10 & 1 & 351683046 & 59520825 & \\
\hline 10 & 2 & 792534015 & & \\
\hline 10 & 3 & 351683046 & & \\
\hline 10 & & 1495900107 & 59520825 & \\
\hline 11 & 1 & 7034538511 & 4304016990 & \\
\hline 11 & 2 & 26225260226 & 4304016990 & \\
\hline 11 & 3 & 26225260226 & & \\
\hline 11 & 4 & 7034538511 & & \\
\hline 11 & & 66519597474 & 8608033980 & \\
\hline 12 & 1 & 111159740692 & 158959754226 & 24325703325 \\
\hline 12 & 2 & 600398249550 & 354949166565 & \\
\hline 12 & 3 & 993494827480 & 158959754226 & \\
\hline 12 & 4 & 600398249550 & & \\
\hline 12 & 5 & 111159740692 & & \\
\hline 12 & & 2416610807964 & 672868675017 & 24325703325 \\
\hline 13 & 1 & 1480593013900 & 4034735959800 & 2208143028375 \\
\hline 13 & 2 & 10743797911132 & 14805457339920 & 2208143028375 \\
\hline 13 & 3 & 25766235457300 & 14805457339920 & \\
\hline 13 & 4 & 25766235457300 & 4034735959800 & \\
\hline 13 & 5 & 10743797911132 & & \\
\hline 13 & 6 & 1480593013900 & & \\
\hline 13 & & 75981252764664 & 37680386599440 & 4416286056750 \\
\hline 14 & 1 & 17302190625720 & 79553497760100 & 100940771124360 \\
\hline 14 & 2 & 160576594766588 & 420797306522502 & 223790013148500 \\
\hline 14 & 3 & 517592962672296 & 691650582088536 & 100940771124360 \\
\hline 14 & 4 & 750260619502310 & 420797306522502 & \\
\hline 14 & 5 & 517592962672296 & 79553497760100 & \\
\hline 14 & 6 & 160576594766588 & & \\
\hline 14 & 7 & 17302190625720 & & \\
\hline 14 & & 2141204115631518 & 1692352190653740 & 425671555397220 \\
\hline 15 & 1 & 182231849209410 & 1302772718028600 & 3130208769783780 \\
\hline 15 & 2 & 2089035241981688 & 9220982517965400 & 11344028448443832 \\
\hline 15 & 3 & 8615949311310872 & 21853758736216200 & 11344028448443832 \\
\hline 15 & 4 & 16789118602155860 & 21853758736216200 & 3130208769783780 \\
\hline 15 & 5 & 16789118602155860 & 9220982517965400 & \\
\hline 15 & 6 & 8615949311310872 & 1302772718028600 & \\
\hline 15 & 7 & 2089035241981688 & & \\
\hline 15 & 8 & 182231849209410 & & \\
\hline 15 & & 55352670009315660 & 64755027944420400 & 28948474436455224 \\
\hline 16 & 1 & 1763184571730010 & 18475997006212200 & 74520697707149580 \\
\hline 16 & 2 & 24325590127655531 & 166713517116449940 & 387689146050297186 \\
\hline 16 & 3 & 123981042854132536 & 528887751025584600 & 633832536898519848 \\
\hline 16 & 4 & 309197871098871838 & 762684674663536626 & 387689146050297186 \\
\hline 16 & 5 & 415691294404230748 & 528887751025584600 & 74520697707149580 \\
\hline 16 & 6 & 309197871098871838 & 166713517116449940 & \\
\hline 16 & 7 & 123981042854132536 & 18475997006212200 & \\
\hline 16 & 8 & 24325590127655531 & & \\
\hline 16 & 9 & 1763184571730010 & & \\
\hline
\end{tabular}




\begin{tabular}{|c|c|c|c|c|}
\hline$e$ & $v$ & $g=4$ & $g=5$ & $g=6$ \\
\hline 16 & & 1334226671709010578 & 2190839204960030106 & 1558252224413413380 \\
\hline 17 & 1 & 15894791312284170 & 233454817237201560 & 1457897216520222060 \\
\hline 17 & 2 & 258634264294653390 & 2595050050431235488 & 10115530661997850556 \\
\hline 17 & 3 & 1587135819804394530 & 10499075716384241952 & 23746474580826741940 \\
\hline 17 & 4 & 4892650539994184868 & 20269771718252599536 & 23746474580826741940 \\
\hline 17 & 5 & 8419549939292302908 & 20269771718252599536 & 10115530661997850556 \\
\hline 17 & 6 & 8419549939292302908 & 10499075716384241952 & 1457897216520222060 \\
\hline 17 & 7 & 4892650539994184868 & 2595050050431235488 & \\
\hline 17 & 8 & 1587135819804394530 & 233454817237201560 & \\
\hline 17 & 9 & 258634264294653390 & & \\
\hline 17 & 10 & 15894791312284170 & & \\
\hline 17 & & 30347730709395639732 & 67194704604610557072 & 70639804918689629112 \\
\hline 18 & 1 & 134951136993773100 & 2682208751185413450 & 24464684545968004800 \\
\hline 18 & 2 & 2548272396065512974 & 35801820369640556595 & 215810538282954699872 \\
\hline 18 & 3 & 18451302662846918700 & 178505550201444784920 & 675870370096399329024 \\
\hline 18 & 4 & 68503375296263488977 & 439591872915483185214 & 970559177597162956688 \\
\hline 18 & 5 & 145737674581607574840 & 588564117958709029644 & 675870370096399329024 \\
\hline 18 & 6 & 186553519919803261860 & 439591872915483185214 & 215810538282954699872 \\
\hline 18 & 7 & 145737674581607574840 & 178505550201444784920 & 24464684545968004800 \\
\hline 18 & 8 & 3503375296263488977 & 35801820369640556595 & \\
\hline 18 & 9 & 18451302662846918700 & 2682208751185413450 & \\
\hline 18 & 10 & 2548272396065512974 & & \\
\hline 18 & 11 & 134951136993773100 & & \\
\hline 18 & & 657304672067357799042 & 1901727022434216910002 & 2802850363447807024080 \\
\hline 19 & 1 & 1088243826731751690 & 229900 & 40035940 \\
\hline 19 & 2 & 23532893106071038404 & 447016944351510642564 & 3931547761898967889520 \\
\hline 19 & 3 & 197822824662547694148 & 2677324515710001081372 & 15658846910857993085360 \\
\hline 19 & 4 & 866831237081712285138 & 8127109896970086044280 & 30002691954232352277608 \\
\hline 19 & 5 & 2221381417843144801098 & 13881153040572190501512 & 30002691954232352277608 \\
\hline 19 & 6 & 3515647035511186627416 & 13881153040572190501512 & 15658846910857993085360 \\
\hline 19 & 7 & 3515647035511186627416 & 8127109896970086044280 & 3931547761898967889520 \\
\hline 19 & 8 & 2221381417843144801098 & 2677324515710001081372 & 362610922310040035940 \\
\hline 19 & 9 & 866831237081712285138 & 447016944351510642564 & \\
\hline 19 & 10 & 197822824662547694148 & 28449551653853229900 & \\
\hline 19 & 11 & 23532893106071038404 & & \\
\hline 19 & 12 & 1088243826731751690 & & \\
\hline 19 & & 13652607304062788395788 & 50322107898515282999256 & 99911395098598706576856 \\
\hline 20 & 1 & 8391311316938069520 & 281858111998039476900 & 4848655679592076350570 \\
\hline 20 & 2 & 205518653220527665304 & 5131008990500486096250 & 63004600211616713352227 \\
\hline 20 & 3 & 1979281881126113225376 & 36188783145801243558900 & 308528931105126354302392 \\
\hline 20 & 4 & 10071757699155275906824 & 131989618396827099239715 & 751928550048308520251566 \\
\hline 20 & 5 & 30468100266480917147760 & 277921666244135490925320 & 1003326321163364778495004 \\
\hline 20 & 6 & 58089920897558352891672 & 354556747218700475500140 & 751928550048308520251566 \\
\hline 20 & 7 & 71823371612912533887168 & 277921666244135490925320 & 308528931105126354302392 \\
\hline 20 & 8 & 58089920897558352891672 & 131989618396827099239715 & 63004600211616713352227 \\
\hline 20 & 9 & 30468100266480917147760 & 36188783145801243558900 & 4848655679592076350570 \\
\hline 20 & 10 & 10071757699155275906824 & 5131008990500486096250 & \\
\hline 20 & 11 & 1979281881126113225376 & 281858111998039476900 & \\
\hline 20 & 12 & 205518653220527665304 & & \\
\hline 20 & 13 & 8391311316938069520 & & \\
\hline 20 & & 469313030628783700080 & 57582616997225194094310 & 9947795252652107008514 \\
\hline
\end{tabular}




\section{Appendix B}

Polynomial $P_{g}(m, m) /(1-2 m)^{(2 g-2)}$ in the generating function $M_{g}(z)$.

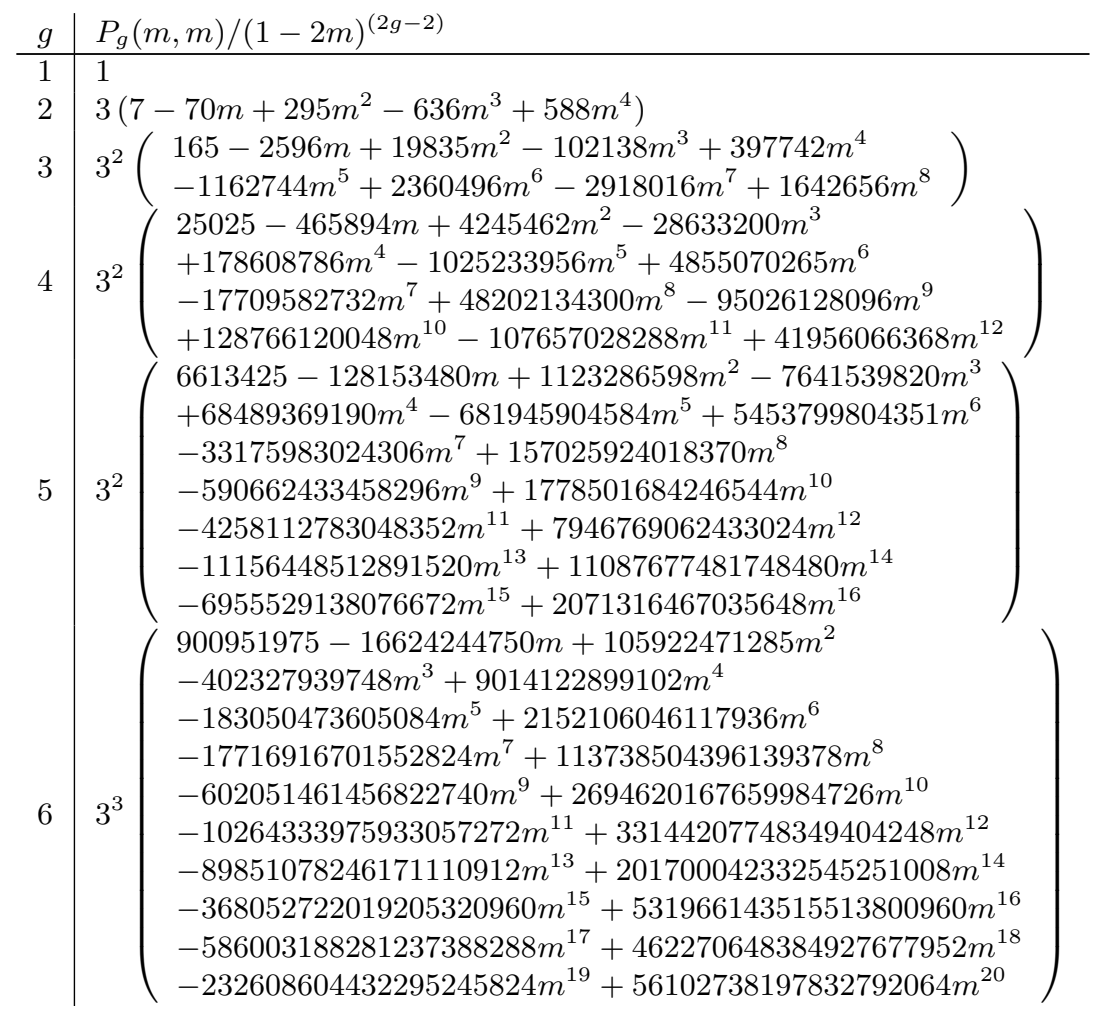

